\title{
Publisher Correction: Gene-based predictive models of trophic modes suggest Asgard archaea are not phagocytotic
}

John A. Burns (D), Alexandros A. Pittis (D) and Eunsoo Kim

Correction to: Nature Ecology \& Evolution https://doi.org/10.1038/s41559-018-0477-7, published online 19 February 2018.

In the version of this Article originally published, question marks appeared in Table 1; they should have been tick marks. This has now been corrected in all versions of the Article.

Published online: 5 March 2018

https://doi.org/10.1038/s41559-018-0520-8 